\title{
Complexity, challenge and creation in curriculum design
}

\author{
S. Mecca ${ }^{1} \&$ J. Thompson ${ }^{2}$ \\ ${ }^{I}$ Department of Engineering-Physics-Systems, Providence College, \\ Providence, RI, USA \\ ${ }^{2}$ Times 2 Academy, One Times Squared Way, Providence, RI, USA
}

\begin{abstract}
Schools today are faced with both the internal and external imposition of standards or benchmarks, which we will term BKs, that are expected to drive curriculum. Benchmarks, such as those of Project 2061 from the American Association for the Advancement of Science, are often structured on multiple levels representing a complex network of standards. Furthermore, schools are expected to demonstrate student accomplishment of benchmarks through effective assessment strategies. The instructional landscape in which curriculum is played out is painted with experienced teachers, who have generally been responsible for working in the context of their own grade and their own classroom with teaching-learning units, projects and activities, which we can term Curriculum Activity Blocks or CABs. CABs have become a part of a teacher's resource inventory, a repertoire so to speak, and while these often address one or more of a school's benchmarks, they are assembled in ways that may not address hierarchical benchmarks in optimum ways, leaving gaps that can result in students being left behind for lack of an adequate curriculum. This paper addresses these issues with both technical and experiential analysis of the complexities and challenges involved in the broad area of curriculum design and documents important fundamental lessons for those who have the responsibility to develop curriculum in the face of multiple demands.
\end{abstract}

\section{Origins and structure of hierarchical standards}

Curriculum in US public education has generally been viewed as specific information that gets taught during a designated period of time. This specific 
information to be taught is often identified through published texts, kits, and programs, or as information that an individual or a group of teachers deem as important for students to learn. From the outside this seems to be a viable method in which to assemble teaching units or curriculum activity blocks, which we will refer to as CABs. After all, teachers have knowledge of national and state standards and publishers often advertise their products as being aligned with national standards. So what's the problem? Consider for a moment that you are a fourth grade teacher and have decided that a CAB on the Rainforest is an appropriate unit of instruction that takes into consideration specific national science and social studies standards as well address pertinent AAAS Project 2061 science literacy benchmarks, and state assessment expectations. You construct your unit to extend for a six-week period of time and even allow for interdisciplinary work with English Language Arts standards through reading about the Rainforest and writing reports on plants and animals in the Rainforest. Now, imagine that one of your colleagues, who teaches second grade also believes that a $\mathrm{CAB}$ on the Rainforest would be appropriate. He too incorporates standards and benchmarks, which we will refer to as BKs, and an interdisciplinary approach to teaching. Each teacher's Rainforest unit has a solid foundation and appears to be developmentally appropriate. The dilemma of relying on teachers working in isolation becomes obvious through the above example as students are exposed to a duplication of information.

If there is a specified period of time (six weeks in the above example during a 36 week school year) in which students are expected to be taught, to learn, and to demonstrate understanding of specific standards and benchmarks, BKs, duplication of information also implies gaps in the teaching and learning of other essential BKs. In addition, the question of when is it developmentally appropriate to teach students the information from the Rainforest unit begs an answer. This duplication / gap issue can be found in all grades and in all subject areas in which a school, district, or county-wide aligned curriculum is nonexistent. Although it is true that publishers do consult national content standards as well as other association benchmarks, there is not necessarily an agreement on which grade should be responsible for teaching the specific BKs. In fact, many publishers advertise their kits or texts to be appropriate for a range of grades. Additionally, not all BKs are addressed in the texts, kits, or publications. Consequently, a reliance on publishers' kits or texts will inherently leave gaps in the critical knowledge base students need for subsequent learning.

Another issue which exists in creating CABs is that some BKs are very broad statements of what students should be able to demonstrate. They are not always broken down into specific performance expectations, thus leaving it up to individual teachers to determine what is appropriate for the students in a specific grade to learn and maybe even within the framework of what the teachers are comfortable teaching. Again, overall this appears to make sense as teachers know students developmental needs better than anyone else. Imagine this time that you are a seventh grade English Language Arts teacher and you are expected to teach students "a wide range of literature from many periods in many genres to build an understanding of the many dimensions (e.g., philosophical, ethical, 
aesthetic) of human experience" [1]. Your least favorite genre to teach is historical fiction so you leave this for last and more often than not, you don't have enough time in the school year to fit in the teaching of this genre. Unfortunately, your colleagues in sixth and eighth grade don't teach historical fiction because they believe that it should be taught in seventh grade. Subsequently, students in your middle school are not taught the dimensions of human experience through historical fiction, thus creating a gap in the teaching / learning process.

The above examples look at the developmental timeline in teaching BKs, but what about conceptual timelines? Is there a hierarchal nature to the teaching of BKs? In other words, does one concept build upon another? Upon examination of the AAAS Project 2061 benchmarks and Atlas concept maps, a complex hierarchy is evident. Specific benchmarks or learning goals are expected to be mastered by the end of grades 2, 5, 8, and 12. Students in grade nine may struggle with understanding specific learning goals or concepts if connected learning goals have not been taught in the previous grades through an organized, cohesive and spiraling curriculum. See Figure 1. What then happens to the learning cycle of these struggling students? An astute teacher would be able to reflect upon where the gap(s) are for the student and backward map their instruction. But more often than not, students are expected to know specific information and concepts prior to being exposed to new information and teachers have limited time in which to teach their own coursework. Consequently, this notion of conceptual support does not occur, especially when teachers are unsure what specific information students were exposed to in previous grades or teachers may not be knowledgeable of the precursors to the learning concepts. To complicate matters more in the creation of a curriculum, AAAS Project 2061 "softens the boundaries between traditional subject areas" [3]. Typical high school science courses include biology, chemistry, physics, geology, astronomy, etc. However Project 2061 benchmarks describes what students should know about within the physical setting and in the living environment, but not explicitly within the typical high school coursework, rather in a cross-grade and crosssubject manner. Project 2061 benchmarks also expect students to be aware of the similarities between the natural and social sciences and to learn about the interdependencies of science, mathematics, and technology.

A carefully planned curriculum will not guarantee that all students will master content specific concepts, however it will provide a resource for teachers to refer to in order to know the learning goals of prior grade levels to allow for the support of student learning.

Standards and benchmarks, BKs, are not a curriculum document. They specify for teachers, administrators, and central office personnel the essential knowledge, skills, and habits of mind that should be taught and learned by students as they progress through their schooling. BKs are the necessary building blocks for the design of a cohesive and aligned curriculum. But which BKs should be addressed when designing curriculum? Each specific discipline has national standards and/or benchmarks, and states have their own standards. 


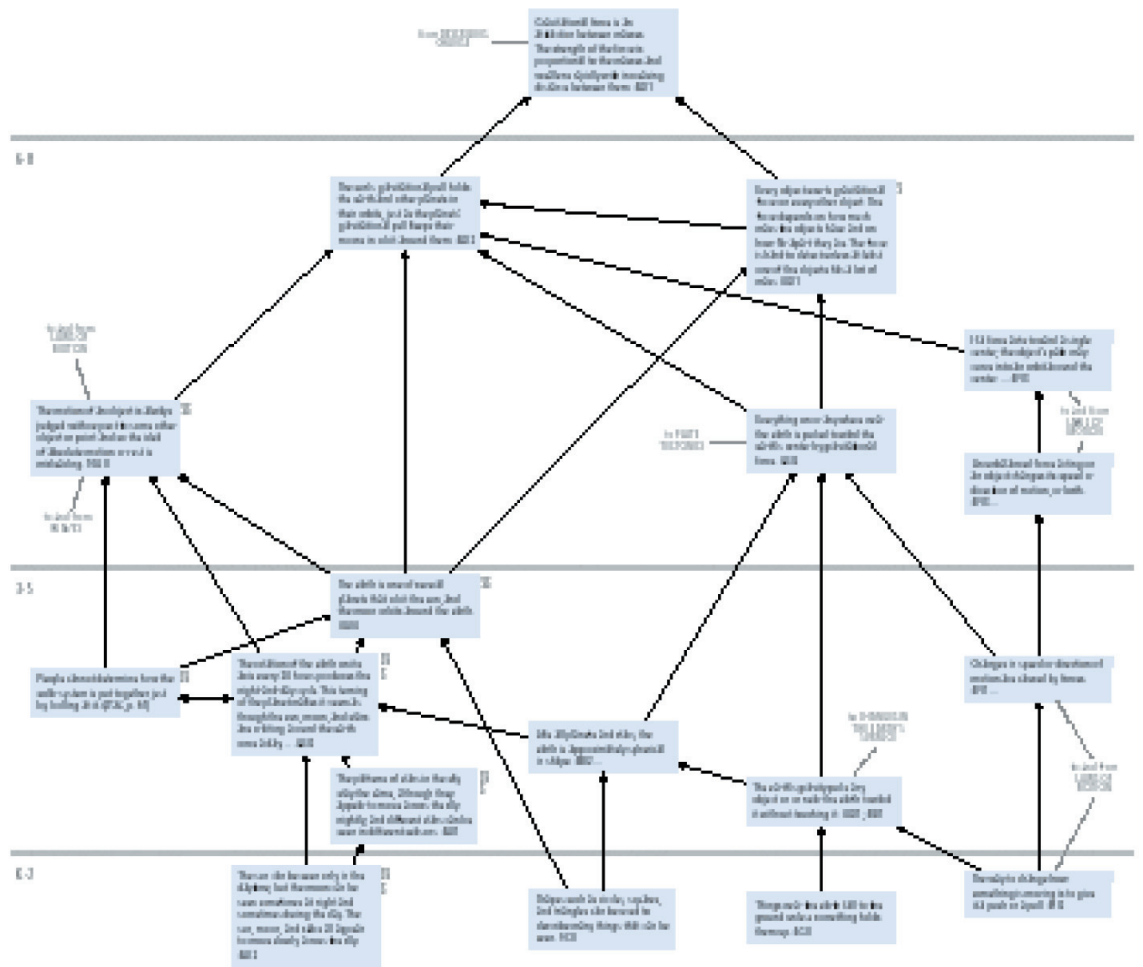

(a)

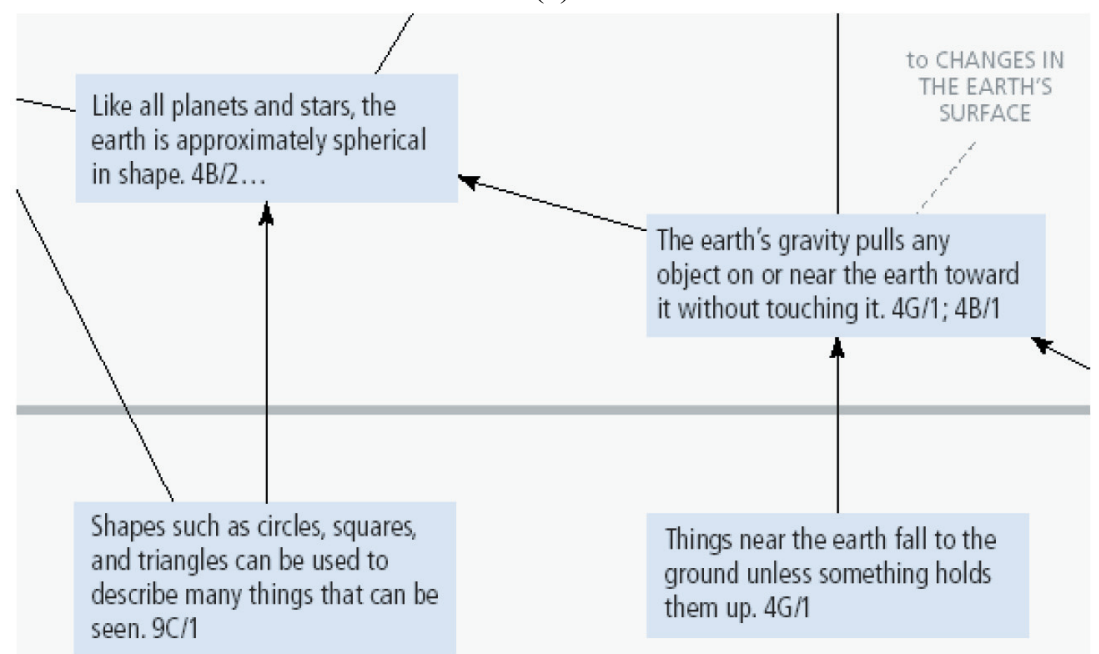

(b)

Figure 1: (a) Excerpt from AAAS Atlas of Science Literacy Processes. (b) Enlargement of (a) lower center section. 
Some are hierarchal, some are grade span specific, some are grade level specific, and others are broad-based learning goals. Putting the pieces together can be a monumental task. In addition, state education departments and local education departments assess student learning of the standards through mandated assessment programs, thus making it even more critical for schools and local districts to put the pieces together with a cohesive curriculum.

\section{Curriculum design by assembly as an optimization process}

The process of coupling CABs together to form a 'curriculum', reflecting the constraints of time, schedule and the context of a school year, has been termed 'design by assembly'. The process has been briefly described in an earlier paper [3] and more extensively in a publication of the AAAS [4].

As noted earlier, teachers placed in classroom settings often confront their curriculum issues with autonomy. Their process begins with a personal inventory of CABs drawn from their experience, a textbook series and/or the collective wisdom of the teaching team with whom they are working. The value of a $\mathrm{CAB}$ is often gleaned from how prior students responded to the activity. Benchmarks are considered by an a priori judgement of which BKs fit a particular $\mathrm{CAB}$ and then the selected $\mathrm{CABs}$ are ordered according to the constraints of time and judgement based on past experience. This process, which we term, 'business as usual', and the resulting curriculum developed using the previously referenced Design by Assembly will create gaps in student learning resulting from a failure to consider benchmarks in their hierarchical order. That is, one will invariably be dealing with $\mathrm{BKs}$ for which the pre-requisite ones have not been accomplished.

To demonstrate this important lesson consider the problem of finding the best order of a given set of CABs to accomplish the included BKs. As an optimization problem, this can be studied by defining a simple scoring algorithm. The process is shown in Figure 2. For each CAB, one arranges the BKs it addresses in best order starting with BKs that are lowest in the network hierarchy. Then, for a given order of CABs, which constitute a curriculum, one has a resulting order of $\mathrm{BKs}$ for that curriculum. The scoring algorithm considers each curriculum BK in order. If the prerequisite BKs have been met, it receives a score of 1 ; if the prerequisite $B K s$ have not been met, it receives a score of -1 and if the BK has already been considered and received a previous score of 1 , the value for that BK is zero. One then adds the scores for each BK in the curriculum and the resulting score (or weighted score $=$ score/number of unique BKs) is recorded. The curriculum with the highest score is the optimum for the set of given CABs. But there is a problem which is often a difficulty with real optimization problems. The number of curricula that one can generate with n CABs taken one at a time is $n$ !.

To illustrate this situation, a set of 7 CABs designated by a Kindergarten teacher was considered. These $7 \mathrm{CABs}$ addressed $63 \mathrm{BKs}$ of which 36 were unique meaning that many BKs were addressed more than once. These units were in actual use in 2003 in a new school that was adopting Project 2061. 
8 The Internet Society II: Advances in Education, Commerce \& Governance

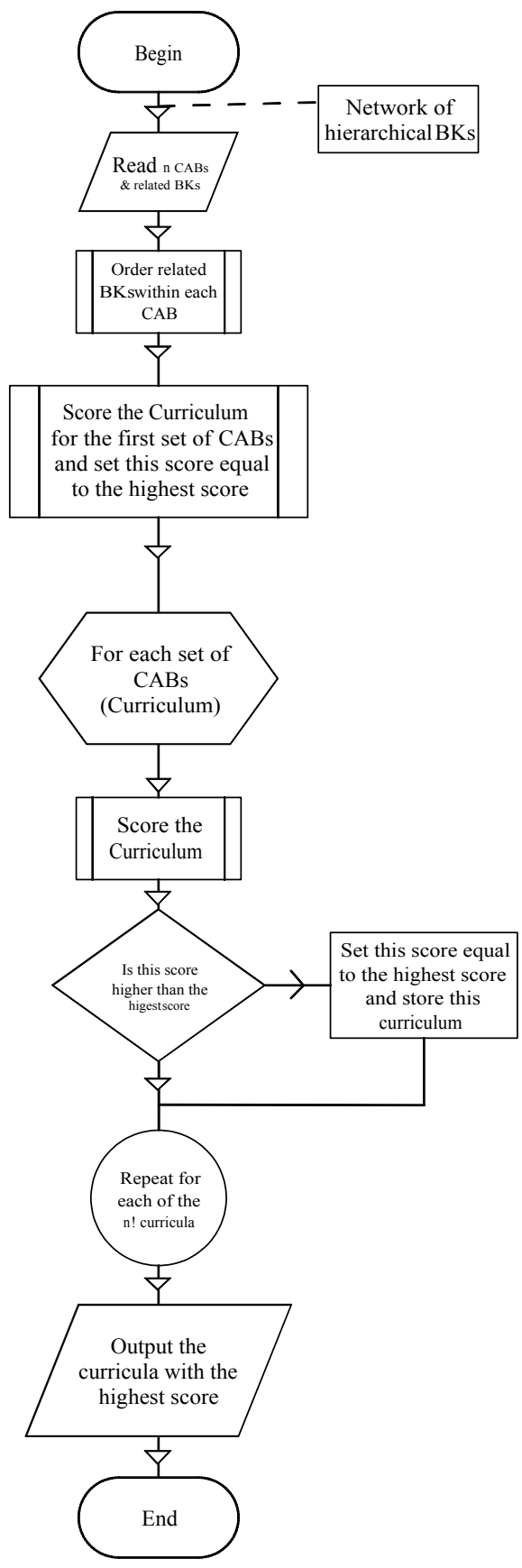

Figure 2: Flowchart representing the search algorithm in block form. See text for the scoring routine. 
Benchmarks as a major part of the framework for its curriculum. These 7 CABs had been assembled into a given order, comprising the Kindergarten curriculum. The first issue here is that there is no simple way to know whether or not this curriculum is optimum for these 7 CABs. There are 7 ! ( 7 factorial) curricula to be 'scored'. On a web-centric system accessing a database for the scoring analyses, it takes about 5 seconds to score a single curriculum (primarily because of the BK hierarchy lookup algorithm). Hence, if one were to do the optimization brute force, the time required would be $5 * 7$ ! seconds or some 7 hours. (Parenthetically, some consideration has been given to reducing this time using an evolutionary algorithm, e.g. a Genetic Algorithm or an Annealing Algorithm, especially for problems with a larger number of CABs. Another grade, for example, had $45 \mathrm{CABs}$ spanning the grade. This would result in as many as 45 ! possible curricula solutions. Such a problem even using one of the aforementioned algorithms was considered practically impossible on today's desktop workstations.) One must also consider that some CABs could be done in parallel. Theoretically, a perfect curriculum addressing 36 unique BKs would have a score of 36 or a weighted score of 1 . The maximum score possible from this 7 CAB set would be obtained if one did all 7 CABs in parallel, an unfeasible solution. The resulting score for this curriculum turns out to be 18 or a weighted score of 0.5 , which is half of the maximum for an ideal $36 \mathrm{BK}$ curriculum. So, by design, the $\mathrm{CAB}$ set falls short regardless of how these are assembled into a curriculum. The score for the curriculum reflecting the actual order in which these $7 \mathrm{CABs}$ were used in the class is .47 and while this is close to the maximum possible for this set, clearly one is trying to address many BKs for which the prerequisite BKs have not been met. In fact, even for the optimum curriculum from this set of $\mathrm{CABs}, 13 \mathrm{BKs}$ were attempted without having met the prerequisite ones. The conclusion is that the 'business as usual' approach to curriculum design, beginning with an inventory of CABs will create large gaps in student learning resulting from a failure to consider benchmarks in their hierarchical order. Students who have not been able to acquire these prerequisite elements in some other way will be left behind.

Table 1 shows an excerpt from an overlay of CABs on hierarchical BKs (shown underlined). Notice the gaps in unfilled BKs. This design shortfall will result in shortfalls in student achievement unless the missing standards are addressed.

\section{An alternate strategy}

In 2004, one of the authors (J.T.) led a teacher curriculum group to address the problem of curriculum development by starting with the benchmarks and thoughtfully configuring CABs to address this paying attention in the design to the order of BKs in the network. A compromise had to be made in choosing CABs due to the constraints on available materials. Fourteen Kindergarten CABs were developed, coupled and assembled to form the curriculum. The weighted score for this curriculum is .51. The maximum weighted score for these 14 CABs (14 CABs taken 14 at a time) is .65 and, while the weighted 
results are still far from a perfect curriculum, the CAB set by design is significantly better than the aforementioned set, suggesting the advantages of letting benchmarks drive the development of CABs rather than the 'business as usual' approach. Yet, there is a long way to go and attention must be placed on the development of CABs that address lowest level BKs. The constraint of available unit material must also be addressed.

Table 1: $\quad$ Excerpt of benchmarks with curriculum activity blocks overlayed.

\begin{tabular}{|c|c|c|}
\hline 120402001 & $\frac{010 \mathrm{~B} 02001}{\text { OKSC_DiversityGarden }}$ & $010 \mathrm{~A} 02001$ \\
\hline & $\begin{array}{l}\frac{010 \mathrm{~B} 02003}{\text { OKLS_DiversityLife }} \\
\text { OKLS_Fall } \\
\text { OKSC_Gardening }\end{array}$ & $010 \mathrm{~B} 02004$ \\
\hline \multirow[t]{6}{*}{$120 \mathrm{~B} 0200 \mathrm{~L}$} & $020 \mathrm{CO} 02001$ & \\
\hline & $\frac{090 \mathrm{~A} 0200 \perp}{\text { OKMA_Seedcount }}$ & $020 \mathrm{C} 02001$ \\
\hline & & $080 \mathrm{E} 02002$ \\
\hline & $000 \Delta 02002$ & \\
\hline & $120 \mathrm{~B} 02002$ & $120 \mathrm{~B} 02004$ \\
\hline & $\begin{array}{l}\frac{1200002001}{\text { OKLS_DiversityLife }} \\
\text { OKLS_Fall }\end{array}$ & $\begin{array}{l}\frac{010 \mathrm{~B} 02003}{\text { OKLS_DiversityLife }} \\
\text { OKLS_Fall } \\
\text { OKSC_Gardening }\end{array}$ \\
\hline
\end{tabular}

When one is forced to work with given materials while still trying to design $\mathrm{CABs}$ that address $\mathrm{BKs}$ in appropriate order, the resulting curriculum will require the interweaving of carefully and creatively designed CABs that address prerequisite BKs. One might be tempted to see this process as a 'bend to fit' strategy, but unless a school system is willing to invest in extensive material and resource development, this appears to be the best one can hope for in a very complex situation. To the extent that schools demand from publishers learning materials sequenced around the relational structure of many of today's standards, we can expect future curricula to be free of the gaps that leave students behind. The lessons learned from the aforementioned analysis are fundamental to meaningful reform of the teaching-learning enterprise.

\section{Lessons learned and conclusion}

Robert Marzano [5] ranks a "guaranteed and viable curriculum" as "having the greatest impact on student achievement." Yet, when looking at the above 
concerns, can we ever create a curriculum that allows for all students to be academically successful and meeting grade-level expectations and standards as expected and measured by the No Child Left Behind Act [6]? There is no question in our minds, that a viable and cohesive curriculum, which allows opportunity for all students to learn, must be created in a planned and purposeful manner. If we are in agreement that this curriculum must be in place to allow for increased student achievement, then educators must abandon the "business as usual" philosophy and begin to examine standards and benchmarks which students are expected to master in the following manner.

1. A content curriculum must include standards and benchmarks, which are broken down into specific developmentally appropriate instructional concepts and are taught by all teachers in a particular grade level or content area within the available time frame. The standards and benchmarks must be analyzed for continuity and hierarchy in order to scaffold student understanding. Failing to scaffold the curriculum will once again result in student learning gaps.

2. Teachers must become familiar with the content and instructional concepts that are taught in previous years as well as what students are expected to know in the upcoming years in order to fill in any gaps that may have occurred and to be sure that the teaching is moving in the direction of the expected curriculum. In addition, teachers, administrators, parents, and central office personnel must know what content is essential for all students and what information can be considered as supplemental.

3. Teacher favored units or CABs will need to be either redesigned so that they meet the expected curriculum or they will need to be abandoned altogether. Existing CABs cannot be shoehorned to fit into a curriculum that is structured in a cohesive or interconnected manner. The teacher developed CABs cannot be haphazard or based on teacher comfort level or interest and they should not have the option to discount the identified curriculum. This teacher implemented curriculum would again, break down the essential standards and benchmarks necessary for the curriculum to be optimal.

The intent of a cohesive and spiraling curriculum is not to provide a day-today lesson plan or a scope and sequence for teachers to follow. This type of curriculum would still allow for and indeed encourage teacher creativity and various methodologies of implementation. Rather, the rationale behind a planned and purposeful curriculum is to provide all students with the opportunity to learn strategies, information, and the problem-solving skills necessary in becoming successful and contributing members of the larger community. If the curriculum is left up to well-intentioned publishers and teachers there will be huge gaps in student learning and this should not be an option for the students in our schools. 
This paper has examined the complexity surrounding teaching and learning as it relates to student achievement with standards and hierarchical benchmarks arising from state, federal and the best thinking of associations entrusted with defining what students should know as a result of their K-12 pre-college experience. The 'business as usual' approach to curriculum development involving 'design by assembly' of an inventory of teaching-learning activities, CABs addressing one or more of the aforementioned benchmarks is shown to be ineffective in meeting this challenge and will invariably leave children behind as a result of the presence of inadvertent gaps in the learning stream. The authors propose a new paradigm for curriculum design, which we term 'design by creation', a process that begins, not with CABs but with the benchmarks proposed to drive the K-12 experience. Design by creation should drive the curriculum development process in an iterative way reflecting the continuing availability of materials and resources that are structured to meet hierarchical standards against which the success of our educational missions are judged. If this form of creationism is taken seriously, teaching and learning will take on a new and exciting vitality, one that is purposeful in intent, results oriented, reflective of new educational technologies and, most importantly, remarkable in student performance.

\section{Acknowledgements}

The authors wish to thank Gail Thomas for her efforts in supporting the IT needs underlying this paper including gleaning required data from TraxLiteracy.com's database and coding the necessary routines to score the curricula described herein. One of the authors (SM) expresses his gratitude to Maria C. Cunha of the University of Coimbra for her patience in explaining the nuances of the Annealing Algorithm and its potential applicability to the optimization problem described in this paper.

\section{References}

[1] Standards for the English Language Arts, National Council of Teachers of English and International Reading Association (1996).

[2] Atlas of Science Literacy Processes, AAAS and the National Science Teachers Association, Washington, D.C., Oxford University Press (2001).

[3] Andrew Ahlgren and Sofia Kesidou, Toward a Coherent Curriculum, ASCD, (1995).

[4] Designs for Science Literacy, AAAS, Washington, DC, Oxford University Press 2000.

[5] Robert J. Marzano, What Works in Schools: Translating Research into Action, ASCD, 2003.

[6] U.S. Department of Education (2001). No Child Left Behind Act, Public Law 107-110. 